Dolichomitus kriechbaumeri (Schulz) (Hymenoptera: Ichneumonidae)'nin Sphenoptera (Tropeopeltis) tappesi Marseul, (Coleoptera: Buprestidae) Üzerindeki Popülasyon Gelişimi

\author{
Halil BOLU ${ }^{1}$, Hasan MARAL ${ }^{2 *}$
}

ÖZET: Bu çalışma, Diyarbakır ilinde 2007-2008 yıllarında; kayısı, şeftali, erik ve kiraz türlerinden kurulmuş deneme bahçesinde, Sphenoptera (Tropeopeltis) tappesi Marseul (Coleoptera: Buprestidae)'un larva parazitoidi Dolichomitus kriechbaumeri (Schulz) (Hymenoptera: Ichneumonidae)'nin populasyon yoğunluğunun belirlenmesi amacıyla yapılmıştır. Meyve ağaçlarının $S$. tappesi'nin larvaları ile bulaşık bitki kısımları Ekim-Kasım aylarında kök kısımları dâhil olmak üzere sökülerek laboratuvara getirilerek ayrı ayrı kültüre alınmış ve hem zararlı hem de parazitoidin ergin çıkışı beklenmiştir. Çalışma sonucunda parazitoid yoğunluğu şeftali, erik, kiraz ve kayısı çeşitlerinde sirasiyla 274, 102, 26 ve 25 adet olarak tespit edilmiştir. En fazla parazitoid sayısı 86 bireyle Cherokee (Şeftali) çeşidinden elde edilmiştir. Bitki aksamlarındaki yoğunluk incelendiğinde en yüksek yoğunluk 134 bireyle şeftalinin gövdesinde görülmüştür. Parazitoid:zararlı oranı kiraz, şeftali, erik ve kayısıda sırasıyla 3.71, 1.67, 0.40 ve 0.29 olarak hesaplanmıştır. Sonuç olarak D. kriechbaumeri'nin GAP illeri meyve alanlarında mücadelesi oldukça zor olan $S$. tappesi'nin kontrolünde önemli bir doğal düşman olduğu, bu nedenle yayılışının dikkatle izlenmesi ve korunması gerektiği sonucuna varılmıştır.

Anahtar Kelimeler: Sphenoptera tappesi, Dolichomitus kriechbaumeri, meyve ağaçları, popülasyon yoğunluğu.

\title{
Population Density of Dolichomitus kriechbaumeri (Schulz) (Hymenoptera: Ichneumonidae) on Sphenoptera (Tropeopeltis) tappesi Marseul (Coleoptera: Buprestidae)
}

\begin{abstract}
This study was conducted to determine the population development of parasitoid Dolichomitus kriechbaumeri (Schulz), larvae parasitoid of Sphenoptera (Tropeopeltis) tappesi Marseul, in the experimental orchard which contains apricot, peach, plum and cherry trees between 2007 and 2008 in Diyarbakır province. Plant parts contaminated with $S$. tappesi larvae and its parasitoid $D$. kriechbaumeri were brought to laboratory and cultured separately. As a result of the study, the parasitoid density was determined as 274, 102, 26 and 25 on peach, plum, cherry, and apricot respectively. The highest parasitoid number was obtained from the Cherokee (Peach) cultivar with 86 individuals. The highest density was observed in the trunk of peach trees with 134 individuals. Parasitoid:pest ratio was calculated as $3.71,1.67,0.40$ and 0.29 on cherry, peach, plum, and apricot. It is concluded that $D$. kriechbaumeri is an important natural enemy of $S$. tappesi, which is very difficult to control in the fruit orchards of the GAP provinces, and therefore its spread should be carefully monitored and protected.
\end{abstract}

Keywords: Sphenoptera tappesi, Dolichomitus kriechbaumeri, fruit trees, population density.

\footnotetext{
${ }^{1}$ Halil BOLU (Orcid ID: 0000-0001-5488-0056), Dicle Üniversitesi Ziraat Fakültesi, Bitki Koruma Bölümü, Diyarbakır, Türkiye

${ }^{2}$ Hasan MARAL (Orcid ID: 0000-0001-9201-8758), Karacadağ Kalkınma Ajansı, Diyarbakır, Türkiye

*Sorumlu Yazar/Corresponding Author: Hasan MARAL, e-mail: hasanmaral@ hotmail.com

Makale 03-05 Şubat 2014 tarihlerinde Antalya'da düzenlenen “Türkiye V. Bitki Koruma Kongresi’nde” poster olarak sunulmuştur.
}

Geliş tarihi / Received: 30-10-2019

Kabul tarihi / Accepted: 01-02-2020 


\section{GíRiș}

Türkiye sahip olduğu iklim koşulları ve toprak yapısı nedeniyle dünyada meyvecilikte söz sahibi ülkelerden biridir. Hem endüstride hem de sofralarda sıklıkla tercih edilen sert çekirdekli meyveler grubu ülkemiz meyve üretiminin \%20'sini oluştururken, bu oranın içerisinde en büyük payı \%59 ile kayıs1, şeftali, erik ve kiraz almaktadır (Anonim, 2018). Dünya toplam meyve üretimi içerisinde Türkiye, kirazda $\% 22$, kayısıda $\% 20$, şeftalide $\% 3$ ve erikte \%2,6'lık bir paya sahiptir (Anonymous, 2017).

Sert çekirdekli meyvelerde verim ve kalite kayıplarına neden olan bir çok zararlı tür mevcuttur. Bu zararlı türlerin bir kısmını barındıran Sphenoptera Dejean (Coleptera: Buprestidae) cinsi türleri sıcak ve kurak bölgelerde meyve ağaçlarının kök, gövde ve yapraklarında önemli zararlara neden olmaktadır (Feng ve ark., 2018). Dünyada 1300'ü aşkın türü bulunan bu cinse ait Palaearktik, Afrotropikal ve Oriental bölgelerde 1000'den fazla tür bulunduğu bildirilmiştir (Bellamy 1985, Niehuis ve Tezcan 1993). Türkiye, Sphenoptera faunası bakımından çok zengin bir ülkedir. Yurdumuzda 64'ü endemik olmak üzere 112 kadar tür bulunmaktadır (Lodos ve Tezcan 1995). Bu cinse ait önemli zararlılardan biri olan ve sert çekirdekli meyvelere önemli zararlar veren Sphenoptera (Tropeopeltis) tappesi Marseul, 1865 (Coleoptera: Buprestidae)'nin Arnavutluk, İran, İsrail, Kıbrıs, Makedonya, Mısır, Suriye, Türkiye, Ürdün ve Yunanistan'da yayılış gösterdiği belirtilmiştir (Balachowsky ve ark., 1962; Georghiou, 1977; Lodos ve Tezcan, 1995; Bolu ve ark., 2005; Kalashian ve Sakalian, 2007; Bolu ve ark., 2011). Zararlının ülkemizde Diyarbakır'da badem kayısı, şeftali, erik ve kiraz ağaçlarında zarar yaptığı, zararlının larvalarının meyve ağaçlarının gövde, anadal ve yandallarında beslenerek zarar neden olduğu ve zararın özellikle yeni kurulan genç meyve bahçelerinde daha fazla olduğu tespit edilmiştir (Bolu ve ark., 2005; Bolu ve ark., 2011).

Sphenoptera cinsine ait türler larvalarının yaşayış şekilleri nedeniyle mücadelesi en zor gruplardan birisini oluştururlar. $\mathrm{Bu}$ nedenle bu cinse ait türlerin doğal düşmanları ve bu doğal düşmanların etkinliklerinin belirlenmesi büyük önem arz etmektedir. Literatürde bu zararlıların doğal düşmanlarına ilişkin sınırlı bilgiler mevcuttur. Waterson (1926), bazı Trichogrammatid (Hymenoptera: Chalcidoidea) türlerinin; Yang ve ark. (2008), bazı Pteraomalid (Hymenoptera: Chalcidoidea) türlerinin Sphenoptera sp.'nin parazitoidi olduğunu tespit etmiştir. Bolu (2008), Dolichomitus kriechbaumeri (Schulz) (Hymenoptera: Ichneumonidae)'nin; Bolu ve ark. (2009) Atanycolus ivanowi (Kokujev) (Hymenoptera: Braconidae)'nin S. tappesi'nin parazitoidi olduğunu bildirmişlerdir.

$\mathrm{Bu}$ çalışma ile S. tappesi'nin larva parazitoidi Dolichomitus kriechbaumeri'nin populasyon yoğunluğunun belirlenmesi amaçlanmıştır.

\section{MATERYAL VE YÖNTEM}

Çalışma 2007-2008 yıllarından Dicle Üniversitesi Ziraat Fakültesi Bahçe Bitkileri Bölümüne ait deneme bahçesinde yürütülmüştür. Bahçe 2002 yılında tesis edilmiş olup, bu çalışmada kayısı (Prunus armeniaca L.) (Aprikoz, Hacıhaliloğlu, Soğancı ve Şekerpare çeşitleri), şeftali [P. persica (L.) Batsch] (Dixired, Earlyred, Cherokee ve Indenpendence çeşitleri), erik (P. cerasifera Ehrh.) (Papaz ve Can çeşitleri), Japon eriği ( $P$. salicina Lindl.) (Santarosa çeşidi) ve kiraz ( $P$. avium L.) (0900-Ziraat ve Napolyon çeşitleri) ağaçları kullanılmıştır. Her çeşit 40'ar ağaçlık parsellerden oluşmuştur.

Sphenoptera (Tropeopeltis) tappesi larvaları ile doğal olarak bulaşı olan bahçede, yapılan gözlemler ile ağaçların gövde ve dallarının kabuk kısmında, kavlama, çatlama ve zamk akıntısı belirtileri görülen, kurumuş veya kurumaya yüz tutmuş ağaçlar tespit edilmiştir. Bu belirtileri gösteren ağaçların zararlı ile bulaşık olabileceği kabul edilmiştir. Zararlı ile bulaşık olarak kabul edilen 69 ağaç 2007 yılının Ekim-Kasım aylarında kök kısımları dâhil olmak üzere sökülerek laboratuvara getirilmiştir. Kök, gövde, 
anadal ve yandal olmak üzere kısımlara ayrılan ve kesilerek parçalanan ağaçlar 30X30X30 ebatlarındaki plastik kaplara konularak, kapların üzeri sık dokunmuş bez ile kapatılmıştır. Parazitoid:zararlı oranının hesabında her bir ağaç üzerinde bulunan toplam parazitoidler sayılmış ve aynı ağaç üzerinde bulunan toplam zararlı sayısına oranlanmıştır.

$\mathrm{Bu}$ işlemler her meyve çeşidi ve ağaç kısımları için ayrı ayrı yapılmıştır. Daha sonra bu kaplar, $26 \pm 1{ }^{\circ} \mathrm{C}, \% 65 \pm 5$ nem ve $16: 8$ aydınlık/karanlık periyoduna ayarlı iklim odasına alınmıştır. 2008 yılının mart ayından itibaren ergin çıkışları başlamıştır. Haziran ayına kadar çıkışlar devam etmiştir. Ergin çıkışları tamamlandığında kültür kaplarından örnekler toplanarak usulüne göre etiketlenmiş ve kaydedilmiştir. Böylece kültüre alınan ağaç kısımlarından zararlı ve parazitoidlerin ergin dönemleri elde edilmiştir.

Zararlının teşhisi, Sayın Prof. Dr. Göksel TOZLU (Atatürk Üniversitesi, Ziraat Fakültesi, Bitki Koruma Bölümü, Erzurum) ve parazitoidin teşhisi Sayın Dr. Yasemin ÖZDEMİR (Ankara Zirai Mücadele Merkez Araştırma Enstitüsü Müdürlüğü) tarafindan yapılmıştır.

\section{BULGULAR VE TARTIŞMA}

Çalışma sonucunda kayısı, şeftali, erik ve kiraz ağaçlarının gövde, anadal ve yandallarında zarar yapan S. tappesi (Şekil 1) ve parazitoidi D. kriechbaumeri'nin (Şekil 2) ergin sayıları Çizelge 1'de verilmiştir.

Çizelge 1. Sphenoptera tappesi (Z) ve Dolichomitus kriechbaumeri (P)'nin değişik meyve ağaçları ve çeşitlerinden elde edilen ergin sayıları

\begin{tabular}{|c|c|c|c|c|c|c|c|c|c|}
\hline \multirow{2}{*}{ Tür } & \multirow{2}{*}{ Çeşitler } & \multicolumn{2}{|c|}{ Gövde } & \multicolumn{2}{|c|}{ Anadal } & \multicolumn{2}{|c|}{ Yandal } & \multicolumn{2}{|c|}{ Toplam } \\
\hline & & $\mathrm{Z}$ & $\mathrm{P}$ & $\mathrm{Z}$ & $\mathrm{P}$ & $\mathrm{Z}$ & $\mathrm{P}$ & $\mathrm{Z}$ & $\mathrm{P}$ \\
\hline \multirow{8}{*}{$\begin{array}{l}\text { 竞 } \\
\text { 可 }\end{array}$} & Can & 16 & $2 q 10^{\pi}$ & 45 & $1196{ }^{\lambda}$ & 15 & 3 3웃 & 76 & $16+12 \pi$ \\
\hline & Formosa & - & - & - & - & - & - & - & - \\
\hline & Giant & 37 & $11+6{ }^{\lambda}$ & - & - & - & - & 37 & $11+6{ }^{\lambda}$ \\
\hline & Papaz & 27 & 10 우 & 9 & 5 우수 & 2 & $5+30$ & 38 & $20 \bigcirc 5 \lesssim$ \\
\hline & President & - & - & 59 & $9+14{ }^{\lambda}$ & 9 & 2 옹 & 68 & $11+19 \lesssim$ \\
\hline & Santarosa & 67 & $10^{\pi}$ & 7 & $1+$ & 10 & - & 84 & 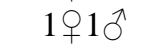 \\
\hline & Stanley & - & - & - & - & - & - & - & - \\
\hline & Total & 147 & $\mathbf{2 3} \odot \mathbf{8} \overbrace{}^{\lambda}$ & 120 & $\mathbf{2 6} \bigcirc \mathbf{2 2} \bigcirc^{\lambda}$ & 36 & $10+13 \overbrace{}^{\lambda}$ & 303 & $\mathbf{5 9} \bigcirc \mathbf{4 3} \hat{\jmath}$ \\
\hline \multirow{7}{*}{ 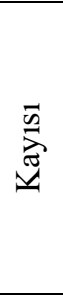 } & Aprikoz & 7 & 79 & 10 & $393 \sigma^{\lambda}$ & - & - & 17 & $10 \bigcirc 3 \sigma^{\lambda}$ \\
\hline & Hacıhaliloğlu & 9 & 1 우 & 3 & - & - & - & 12 & 1 우 \\
\hline & Roxana & 7 & 2 우 & - & - & - & - & 7 & 29 \\
\hline & Soğancı & 1 & - & 8 & 1 우 & - & - & 14 & 1 우 \\
\hline & Şekerpare & 8 & $4 \bigcirc 3 \hat{\jmath}$ & 16 & 1 ㅇ & 12 & - & 36 & 5 웅 \\
\hline & Thirynte & - & - & - & - & - & - & - & - \\
\hline & Total & 32 & $14 \bigcirc 3{ }^{\lambda}$ & 37 & $\mathbf{5} \bigcirc \mathbf{3} \overbrace{}^{\lambda}$ & 17 & - & 86 & $19 \bigcirc 6 \overbrace{}^{\lambda}$ \\
\hline \multirow{6}{*}{$\underset{\mathbb{Z}}{\mathbb{Z}}$} & Lambert & - & - & - & - & - & - & - & - \\
\hline & Napolyon & - & - & - & - & - & - & - & - \\
\hline & Starks Gold & - & $1{ }^{\lambda}$ & - & - & - & - & - & $1 \hat{\sigma}-$ \\
\hline & Van & - & $1 \delta^{\lambda}$ & - & & - & & - & $10^{\pi}$ \\
\hline & 0900-Ziraat & 7 & $15 \bigcirc 9 \widehat{0}$ & - & - & - & - & 7 & $15 \bigcirc 9 ð$ \\
\hline & Total & 7 & $\mathbf{1 5}+11 \overbrace{}^{\lambda}$ & - & - & - & - & 7 & $15+11{ }^{\lambda}$ \\
\hline \multirow{5}{*}{ 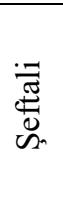 } & Cherokee & 19 & 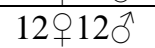 & 12 & $29+20{ }^{\lambda}$ & 9 & $3+10{ }^{\lambda}$ & 40 & $44 \bigcirc 42 \sigma^{\lambda}$ \\
\hline & Dixired & 21 & $17 ㅇ ㅜ 12 \hat{\jmath}$ & 30 & 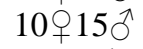 & 9 & $6+7 \lesssim$ & 60 & $33 ㅇ+34$ ○ \\
\hline & Earlyred & 28 & $29+24 \hat{0}$ & 5 & 2070 & 4 & 10 & 37 & $31+32 \widehat{0}$ \\
\hline & Independence & 20 & $16+12 \widehat{\jmath}$ & 5 & $9+18{ }^{\wedge}$ & 2 & $120{ }^{\wedge}$ & 27 & $26 \bigcirc 32 \widehat{ }$ \\
\hline & Total & 88 & $74+60 \AA$ & 52 & $50 \bigcirc 60 \curvearrowright$ & 24 & $\mathbf{1 0}+\mathbf{2 0}$ & 164 & $134+140$ \\
\hline \multicolumn{2}{|c|}{ Genel Toplam } & 274 & $126 \bigcirc 82{ }^{\lambda}$ & 209 & $\mathbf{8 1} \bigcirc \mathbf{8 5} \overbrace{}^{\lambda}$ & 77 & $\mathbf{2 0} \bigcirc \mathbf{3 3} \bigcirc^{\lambda}$ & 560 & $\mathbf{2 2 7} \bigcirc \mathbf{2 0 0} \overbrace{}^{\lambda}$ \\
\hline
\end{tabular}


Çizelge 1 incelendiğinde, en yüksek parazitoid yoğunluğunun şeftalide (134 dişi, 140 erkek), en düşük yoğunluğun ise kayısıda olduğu (19 dişi, 6 erkek) görülmektedir. Bitki aksamlarındaki yoğunluk incelendiğinde en yüksek yoğunluk şeftalinin gövdesinde görülürken (74 dişi, 60 erkek), kayıs1 ve kirazın yan dallarından herhangi bir parazitoid elde edilememiştir.

Çeşitler incelendiğinde en yüksek parazitoid yoğunluğu 86 ergin (44 dişi, 42 erkek) ile Cherokee (Şeftali) çeşidinden elde edilirken, Formosa, Stanley (Erik), Thirynte (Kayısı), Lambert ve Napolyon (Kiraz) çeşitlerinden herhangi bir parazitoit elde edilememiştir.

Ağaçlardan elde edilen parazitoid:zararlı oranı kirazda 3.71, şeftalide 1.67, erikte 0.40 ve kayısıda 0.29 olarak hesaplanmıştır. Tüm araştırmadaki genel oran 0,76 olarak tespit edilmiştir. Parazitoidin eşey oranı yaklaşık $1 / 1$ olarak belirlenmiştir.

Sphenoptera (Tropeopeltis) tappesi meyve ağaçlarının en önemli zararlılarından birisidir (Bolu ve ark. 2005) (Şekil 2). S. tappesi bu kadar önemli bir zararlı olmasına rağmen hem zararlı hakkında hem de larva parazitoidi Dolichomitus kriechbaumeri hakkında literatürde çok az bilgi mevcuttur.

Yu ve ark. (2005), Batı Palearktik bölgede Dolichomitus Smith (Hymenoptera: Ichneumonidae) cinsine ait 27 tür olduğunu, Choi ve ark. (2016), Dolichomitus türlerinin meyve ağaçlarının odun dokularında zarar yapan bir çok coleopter türünün parazitoitidi olduğunu bildirmiştir.

Altay (1966), Cydia molesta (Busck.) (Lepidoptera: Torticidae)'nın Aubert (1969), Anthaxia manca L. (Coleptera: Buprestidae)'nın ve Kalashyan (1984), Anthaxia caucasica Ab. (Coleptera: Buprestidae)'nın D. kriechbaumeri'nin konukçuları arasında yer aldığını tespit etmiştir. Çınar ve ark. (2004) bazı buprestid türlerinin D. kriechbaumeri'nin konukçuları olduğunu bildirmiştir. Zwakhals (2010), D. kriechbaumeri'nin literatürde bazı çalışmalarda belirtildiği gibi Cerambycidae (Coleptera) larvalarına değil, Buprestidae larvalarına özelleşmiş bir parazitoid olma ihtimalinin daha yüksek olduğunu belirtmiştir.

Kishi (1970), Dolichomitus sp.'nin Curculionidae (Coleoptera) familyasına bağlı değişik konukçular üzerindeki eşey oranlarını tespit amacıyla bir çalışma yürütmüş ve çalışma sonucunda Pissodes nitidus Roel. üzerinden elde 42 bireyin tümünün erkek olduğunu, Shirahoshizo spp. üzerinden elde edilen 90 bireyin 8'inin dişi, 82'sinin erkek olduğunu ve Niphades variegatus Roel. üzerinden elde edilen 93 bireyin 54'ünün dişi 39'unun erkek olduğunu tespit etmiştir.
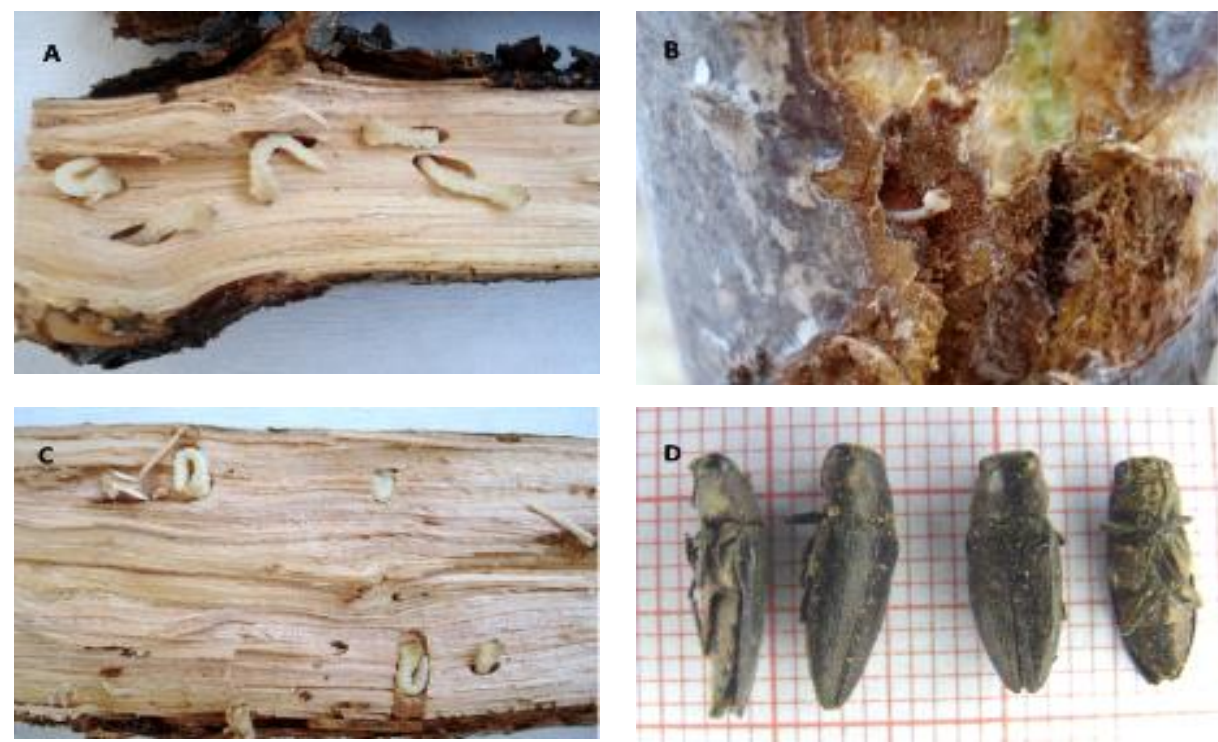

Şekil 1. Sphenoptera tappesi'nin A-yandal içerisinde beslenen larvaları; B- ağacın gövdesinde beslenen larvası; Cprepupa dönemi; D- ergin dönemi. 

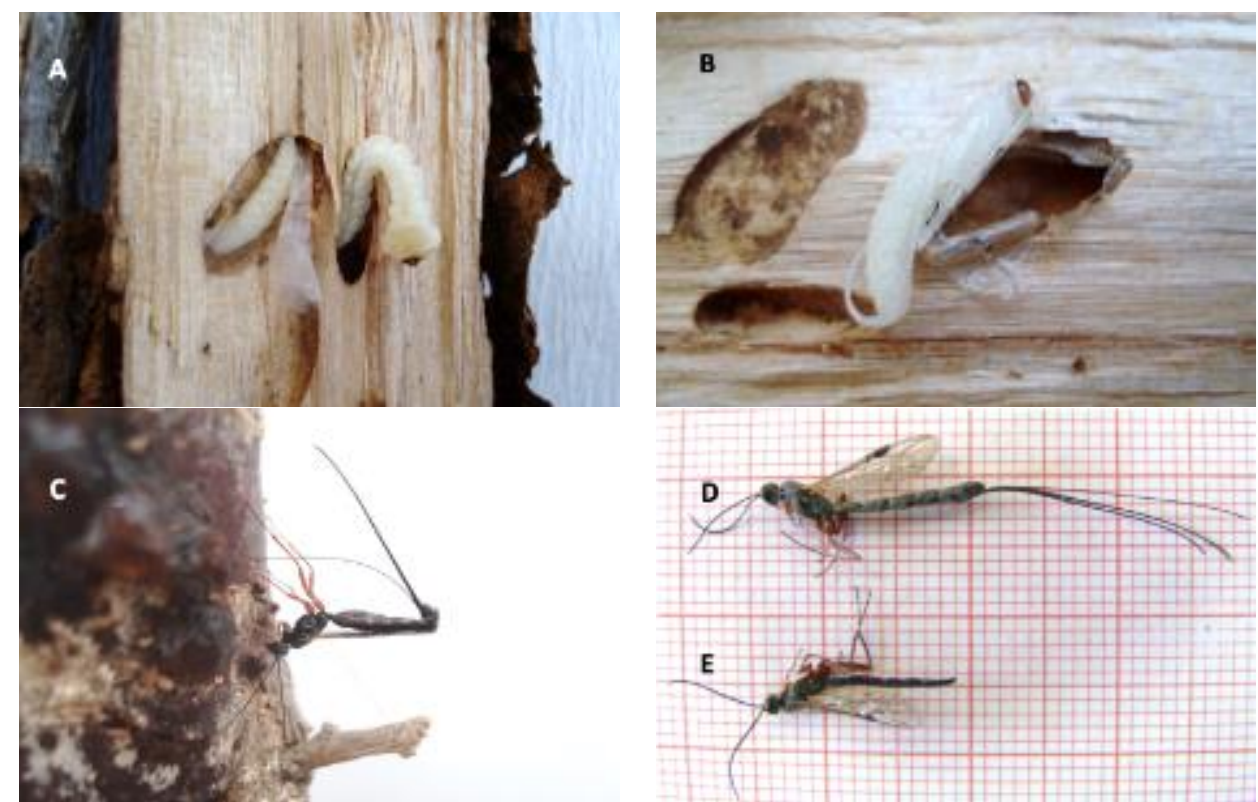

Şekil 2. Dolichomitus kriechbaumeri'nin A- prepupa dönemi (resmin solu); B- pupa dönemi; C- dişisinin gövde içerisindeki Sphenoptera tappesi larvasını parazitleme anı; D- dişisi; E- erkeği.

Bolu (2008), Diyarbakır ilinde erik ve şeftali ağaçlarında zarar yapan S. tappesi'nin dünya için yeni kayıt olan doğal düşmanı D. kriechbaumeri hakkında bilgi vermiştir. Araştırıcı 28 S. tappesi larvasından 4'ü şeftali, 2'si erik ağaçlarından olmak üzere 6 parazitoid elde etmiştir.

\section{SONUÇ}

$\mathrm{Bu}$ çalışma ile meyve ağaçlarının her geçen gün önemi artan zararlılarından birisi olan $S$. tappesi'nin parazitoidi $D$. kriechbaumeri'nin erginlerinin değişik meyve ağaçlarındaki yoğunluğu araştırılmıştır. Parazitoidin etkinliğinde larva sayısından ziyade, eldeki bulgular neticesinde ağacın yapısının önemli olabileceği kanısına varılmıştır. Güneydoğu Anadolu Projesi kapsamında birçok sulama projesi tamamlanmış ve tarlalara su verilmeye başlanmıştır. Bölgede sulama imkanlarının katma değerli üretime dönüştürülmesi amacıyla kapama bahçe kurulumuna yönelik bir çok devlet desteği verilmiş ve bu sayede kurulan bahçe sayısı önemli oranda artış göstermiştir. Bu bahçelerde maksimum verimi elde etmek ve nitelikli üretim yapabilmek için Diyarbakır, Elazığ ve Mardin illeri meyve alanlarında her gün önemi artan ve mücadelesi oldukça zor olan S. tappesi'nin yayılışının dikkatle izlenmesi ve parazitoidi $D$. kriechbaumeri'nin korunması gerekmektedir.

\section{TEŞEKKÜR}

Sphenoptera (Tropeopeltis) tappesi'nin teşhisini yapan Sayın Prof. Dr. Göksel TOZLU'ya (Atatürk Üniversitesi, Ziraat Fakültesi, Biyoloji Bölümü, Erzurum) ve Dolichomitus kriechbaumeri'nin teşhisini yapan Sayın Dr. Yasemin ÖZDEMİR'e (Ankara Zirai Mücadele Merkez Araştırma Enstitüsü Müdürlüğ̈̈) teşekkür ederiz.

\section{KAYNAKLAR}

Anonymous, 2017. FAO, Crop Production Statistics, http://www.fao.org/faostat/en/\#data/QC, (Erişim Tarihi: 26.10.2019).

Anonim, 2018. Tarım ve Orman Bakanlı̆̆1, Bitkisel Üretim İstatistikleri, https://www.tarimorman.gov.tr/sgb/Belgeler/SagMenuVeriler/BUGEM.pdf, (Erişim Tarihi: 26.10.2019).

Altay M, 1966. Investigations on Biology and Control of Laspeyresia molesta Busck in Bursa Province and Marmara Region, Teknik Bulten 1, Yenilik Basimevi, 55 s., İstanbul. 
Aubert JF, 1969. Les Ichneumonides Ouest-Paléarctiques et Leurs Hôtes, Tome, 1: Pimplinae, Xoridinae, Acaenitinae. Laboratoire d'Evolution des Etres Organises, 302 pp., Paris-France.

Feng CY, Zhang L, Li W, Yang X, Zong S, Balachowsky, 2018. Cold Hardiness of Overwintering Larvae of Sphenoptera sp. (Coleoptera: Buprestidae) in Western China. Journal of Economic Entomology, 111(1): 247-251.

Balachowsky A, Davatchi A, Descarpentries A, 1962. Famille des Buprestidae. In: Entomologie Appliquee a L'Agriculture. Tome I, $564 \mathrm{p}$.

Bellamy CL, 1985. A Catalogue of The Higher Taxa of The Family Buprestidae (Coleoptera). Navorsinge van die Nasionale Museum, Bloemfontein, 4 (15): 405-472.

Bolu H, 2008. A New Host Sphenoptera tappesi Marseul (Coleoptera: Buprestidae) for Dolichomitus kriechbaumeri (Schulz) (Hymenoptera: Ichneumonidae) from Turkey. Turkish Journal of Zoology, 32: 225-226.

Bolu H., Ozgen İ, Çınar M, 2005. Dominancy of Insect Families and Species Recorded in Almond Orchards of Turkey. Acta Phytopathologica et Entomologica Hungarica, 40 (1-2): 145-157.

Bolu H, Beyarslan A, Yildırım H, Aktürk Z, 2009. Two New Host Records of Atanycolus ivanowi (Kokujev, 1898) (Hymenoptera: Braconidae) from Turkey. Türkiye Entomoloji Dergisi, 33 (4): 279-287.

Bolu H, Aktürk Z, Yıldırım H, 2011. Diyarbakır ilinde Sphenoptera (Tropeopeltis) tappesi Marseul, 1865 (Coleoptera: Buprestidae)'nin Bazı Meyve Ağaçlarındaki Ergin Yoğunluğunun Tespiti. Bitki Koruma Bülteni, 51(3): 255-266.

Choi JK, Kolaro J, Jeong CJ, Lee JW, 2016. A Taxonomic Review of the Genus Dolichomitus Smith (Hymenoptera: Ichneumonidae: Pimplinae) From South Korea With Descriptions of two New Species. Zootaxa, 4132(2): 235-253.

Çınar M, Çimen İ, Bolu H, 2004. Elazığ ve Mardin İlleri Kiraz Ağaçlarında Zararlı Olan Türler, Doğal Düşmanları ve Önemlileri Üzerindeki Gözlemler. Türkiye Entomoloji Dergisi, 28: 1-11.

Georghiou GP, 1977. The Insects and Mites of Cyprus. Kiphissia, 347 p., Greece- Athens.

Kalashian MY, 1984. Species of Parasites of Armenian Metallic Wood-borers (Coleoptera, Buprestidae). Biological Journal of Armenia, 37: 986-987.

Kalashian MY, Sakalin VP, 2007. A Review of the Genus Sphenoptera Dejean, 1833 (Coleoptera: Buprestidae) of the Balkan Peninsula. Acta Zoologica Bulgarica, 59(1):17-28.

Kishi Y, 1970. Difference in the Sex Ratio of the Pine Bark Weevil Parasite, Dolichomitus sp. (Hymenoptera: Ichneumonidae), Emerging from Different Host Species. Applied Entomology and Zoology, 5(3): 126-132.

Lodos N, Tezcan S, 1995. Türkiye Entomolojisi V Buprestidae (Genel Uygulamalı ve Faunistik). Entomoloji Derneği Yayınları No: 8. Ege Üniversitesi Basımevi, 138 s, Bornova-İzmir.

Niehuis M, Tezcan S, 1993. Beitrag Zur Kenntnis Der Agrilus-arten Der Türkei (Coleoptera: Buprestidae). Mitteilungen des Internationalen Entomologischen Vereins, 18: 1-74.

Waterson J, 1926. On a New Trichogrammatid (Hym., Chalcidoidea) Parasite of the Cotton Stemborer (Sphenoptera sp.). Bulletin of Entomological Research, 16(4): 309-313.

Yang ZQ, Wang XY, Gould JR, Wu H, 2008. Host specificity of Spathius agrili Yang (Hymenoptera: Braconidae), an Important Parasitoid of the Emerald Ash Borer. Biological Control, 47: 216-221.

Yu DS, Van Achterberg C, Horstmann K, 2005. World Ichneumonoidea- Taxonomy, Biology, Morphology and Distribution. Taxapad. Vancouver, Canada.

Zwakhals K, 2010. Identification of Western Palearctic Dolichomitus species (Hymenoptera: Ichneumonidae: Pimplinae). Entomologische Berichten, 70(4): 111-127. 